\title{
Quark-model based study of the triton binding energy
}

\author{
B. Juliá-Díaz, ${ }^{1}$ J. Haidenbauer, ${ }^{2}$ A. Valcarce, ${ }^{1,3}$ and F. Fernández ${ }^{1}$ \\ ${ }^{1}$ Grupo de Física Nuclear, Universidad de Salamanca, E-37008 Salamanca, Spain \\ ${ }^{2}$ Institut für Kernphysik (Theorie), Forschungszentrum Jülich, D-52425 Jülich, Germany \\ ${ }^{3}$ Departamento de Física Teórica, Universidad de Valencia, E-46100 Valencia, Spain
}

(Received 18 September 2001; published 6 February 2002)

\begin{abstract}
The three-nucleon bound state problem is studied employing a nucleon-nucleon potential obtained from a basic quark-quark interaction in a five-channel Faddeev calculation. The obtained triton binding energy is comparable to those predicted by conventional models of the $N N$ force.

DOI: 10.1103/PhysRevC.65.034001

PACS number(s): 12.39.Jh, 21.45.+v, 13.75.Cs, 21.10.Dr
\end{abstract}

\section{INTRODUCTION}

During the last decade the development of quark-model based interactions for the hadronic force has led to nucleonnucleon $(N N)$ potentials that provide a fairly reliable description of the on-shell data. Several models including quark degrees of freedom have been used to study the $N N$ interaction [1] and also the baryon spectra [2]. Among them, the chiral quark cluster model is the only one that pursued a simultaneous understanding of different low-energy phenomena based on a unique quark-quark interaction. This model is able to provide a quantitative description of the $N N$ scattering [3] and bound state problems [4].

Nevertheless, quark-model based $N N$ interactions have not been often used to study few-body systems. There might be two different reasons for that. First of all, most of those interaction models for the two-nucleon system needed to be supplemented with meson-exchange potentials between the baryons to obtain a reasonable description of the experimental data $[5,6]$, losing in this way their quark based character. Second, other quark-model based interactions were primarily designed to describe the baryon spectra [7], but lead to unrealistic results when they are applied to the two-nucleon system [8].

In this work we want to perform a study of the triton bound state making use of a nonlocal $N N$ potential fully derived from quark-quark interactions. The model has been previously utilized for investigations of three-body systems ( $N N N, N N \Delta, N \Delta \Delta$, and $\Delta \Delta \Delta$ ), putting more emphasis on the mass ordering of possible bound states of these systems than on the binding energy values [9]. In the present work the full nonlocal $N N$ potential will be employed as it follows from the application of the resonating group method (RGM) formalism. This method allows, once the Hilbert space for the six-body problem has been fixed, to treat the intercluster dynamics in an exact way. Thereby, nonlocalities are generated, reflecting the internal structure of the nucleon, which translate into specific off-shell properties of the resulting $N N$ potential.

Indeed the relevance and/or necessity of considering the nonlocal parts of $N N$ potentials in realistic interactions is still under debate. Over the past few years several studies have appeared in the literature which stress the potential importance of nonlocal effects for the quantitative understand- ing of few-body observables and, specifically, for the triton binding energy $[10-15]$. However, the majority of these investigations [11-15] explore only nonlocalities arising from the meson-exchange picture of the $N N$ interaction.

The nonlocalities generated in a quark-model derivation of baryonic potentials may play a relevant role for the case of the three-nucleon bound state. It has been argued that the assumptions associated with meson-exchange models sharply limit the nature of the off-shell properties of those potentials, once the on-shell matrix elements are constrained to fit the $N N$ data [16]. Therefore, it is very interesting to investigate the off-shell features of potentials derived from a quark model. Some preliminary studies in this direction have been done in Ref. [10]. However, there only the short-range part of the interaction is obtained by means of quark-model techniques. The intermediate- and long-range parts are described by "standard" meson exchange between baryons. Accordingly, that work allows only very limited conclusions with regard to effects of the quark substructure.

The triton binding energy is obtained from a Faddeev calculation. We restrict ourselves to the standard five-channel case; i.e., we consider only the ${ }^{1} S_{0}$ and ${ }^{3} S_{1}{ }^{-}{ }^{3} D_{1} N N$ partial waves, those which provide the bulk contribution to the three-nucleon binding energy. The three-body Faddeev equations will be solved in momentum space, making use of separable finite-rank expansions of the two-body interactions.

The paper is organized as follows. In Sec. II we introduce the basic quark-quark Hamiltonian and we describe the method to obtain the RGM $N N$ interaction. In Sec. III we provide details about the finite-rank expansions of the quarkmodel based potentials which enter in the Faddeev calculations of the triton binding energy and we present results for the three-body system. Finally, some concluding remarks are provided in Sec. IV.

\section{QUARK-MODEL BASED NN POTENTIAL}

In recent years a chiral quark cluster model for the $N N$ interaction has been developed. This model has been widely described in the literature $[3,4,17,18]$; therefore, we will only briefly summarize here its most relevant aspects. It contains, as a consequence of chiral symmetry breaking, a pseudo- 
TABLE I. Quark-model parameters. The values in brackets are used for a correct description of the deuteron.

\begin{tabular}{lc}
\hline \hline$m_{q}(\mathrm{MeV})$ & 313 \\
$b^{\mathrm{a}}(\mathrm{fm})$ & 0.518 \\
$\alpha_{s}$ & 0.4977 \\
$g_{c h}^{2}$ & $6.60(6.86)$ \\
$m_{S}\left(\mathrm{fm}^{-1}\right)$ & 3.400 \\
$m_{P S}\left(\mathrm{fm}^{-1}\right)$ & 0.70 \\
$\Lambda_{\chi}\left(\mathrm{fm}^{-1}\right)$ & 4.47 \\
\hline
\end{tabular}

$\bar{a} b$ is the parameter of the harmonic oscillator wave function used for each quark $\eta(x)=\left(1 / \pi b^{2}\right)^{(3 / 4)} e^{-\left(x^{2} / 2 b^{2}\right)}$.

scalar and a scalar exchange between constituent quarks coming from the Lagrangian

$$
L_{c h}=g_{c h} F\left(q^{2}\right) \bar{\Psi}\left(\sigma+i \gamma_{5} \vec{\tau} \cdot \vec{\pi}\right) \Psi
$$

where $F\left(q^{2}\right)$ is a monopole form factor:

$$
F\left(q^{2}\right)=\left[\frac{\Lambda_{\chi}^{2}}{\Lambda_{\chi}^{2}+q^{2}}\right]^{1 / 2} .
$$

$\Lambda_{\chi}$ determines the scale of chiral symmetry breaking, being bound between $1 \mathrm{GeV}$ and $600 \mathrm{MeV}$ [19]. The chiral coupling constant $g_{c h}$ is chosen to reproduce the experimental $\pi N N$ coupling constant.

From the above Lagrangian a pseudoscalar $(P S)$ and a scalar $(S)$ potential between quarks can be easily derived in the nonrelativistic approximation:

$$
\begin{gathered}
V_{i j}^{P S}(\vec{q})=-\frac{g_{c h}^{2}}{4 m_{q}^{2}} \frac{\Lambda_{\chi}^{2}}{\Lambda_{\chi}^{2}+q^{2}} \frac{\left(\vec{\sigma}_{i} \cdot \vec{q}\right)\left(\vec{\sigma}_{j} \cdot \vec{q}\right)}{m_{P S}^{2}+q^{2}}\left(\vec{\tau}_{i} \cdot \vec{\tau}_{j}\right) \\
V_{i j}^{S}(\vec{q})=-g_{c h}^{2} \frac{\Lambda_{\chi}^{2}}{\Lambda_{\chi}^{2}+q^{2}} \frac{1}{m_{S}^{2}+q^{2}} .
\end{gathered}
$$

Using the range of values for $\Lambda_{\chi}$ given above yields a $N-\Delta$ mass difference due to the pseudoscalar interaction between 150 and $200 \mathrm{MeV}$. The rest of the mass difference, up to the experimental value, must have its origin in perturbative processes. In the present model, this is taken into account through the one-gluon-exchange potential [20]

$$
\begin{aligned}
V_{i j}^{O G E}(\vec{q})= & \alpha_{s}\left(\vec{\lambda}_{i} \cdot \vec{\lambda}_{j}\right)\left\{\frac{\pi}{q^{2}}-\frac{\pi}{4 m_{q}^{2}}\left[1+\frac{2}{3}\left(\vec{\sigma}_{i} \cdot \vec{\sigma}_{j}\right)\right]\right. \\
& \left.+\frac{\pi}{4 m_{q}^{2}} \frac{[\vec{q} \otimes \vec{q}]^{(2)} \cdot\left[\vec{\sigma}_{i} \otimes \vec{\sigma}_{j}\right]^{(2)}}{q^{2}}\right\},
\end{aligned}
$$

where the $\lambda$ 's are the color Gell-Mann matrices and $\alpha_{s}$ is the strong coupling constant.

For the present study we make use of the nonlocal $N N$ potential derived through a Lippmann-Schwinger formulation of the RGM equations in momentum space [18]. The formulation of the RGM for a system of two baryons $B_{1}$ and $B_{2}$ needs the wave function of the two-baryon system constructed from the one-baryon wave functions. The twobaryon wave function can be written as

$$
\begin{aligned}
\Psi_{B_{1} B_{2}} & =\mathcal{A}\left[\chi(\vec{P}) \Psi_{B_{1} B_{2}}^{S T}\right] \\
& =\mathcal{A}\left[\chi(\vec{P}) \phi_{B_{1}}\left(\vec{p}_{\xi_{B_{1}}}\right) \phi_{B_{2}}\left(\vec{p}_{\xi_{B_{2}}}\right) \chi_{B_{1} B_{2}}^{S T} \xi_{c}\left[2^{3}\right]\right]
\end{aligned}
$$

where $\mathcal{A}$ is the antisymmetrizer of the six-quark system, $\chi(\vec{P})$ is the relative wave function of the two clusters, $\phi_{B_{i}}\left(\vec{p}_{\xi_{B_{i}}}\right)$ is the internal spatial wave function of the baryon $B_{i}$, and $\xi_{B_{i}}$ are the internal coordinates of the three quarks of baryon $B_{i} \cdot \chi_{B_{i} B_{2}}^{S T}$ denotes spin-isospin wave function of the two-baryon system coupled to spin $(S)$ and isospin $(T)$, and,

\begin{tabular}{|c|c|c|c|c|c|}
\hline & & Quark model & Nijm II [22] & Bonn B [21] & Expt. \\
\hline & & Low- & scattering par & & \\
\hline${ }^{1} S_{0}$ & $a_{s}(\mathrm{fm})$ & -23.759 & -23.739 & -23.750 & $-23.74 \pm 0.02$ \\
\hline & $r_{s}(\mathrm{fm})$ & 2.68 & 2.67 & 2.71 & $2.77 \pm 0.05$ \\
\hline${ }^{3} S_{1}$ & $a_{t}(\mathrm{fm})$ & 5.461 & 5.418 & 5.424 & $5.419 \pm 0.007$ \\
\hline & $r_{t}(\mathrm{fm})$ & 1.820 & 1.753 & 1.761 & $1.753 \pm 0.008$ \\
\hline & & & eron properties & & \\
\hline$\epsilon_{d}$ & & -2.2242 & -2.2246 & -2.2246 & -2.224575 \\
\hline$P_{D}$ & & 4.85 & 5.64 & 4.99 & - \\
\hline$Q_{d}$ & & 0.276 & 0.271 & 0.278 & $0.2859 \pm 0.0003$ \\
\hline$A_{S}$ & & 0.891 & 0.8845 & 0.8860 & $0.8846 \pm 0.0009$ \\
\hline$A_{D} /$ & & 0.0257 & 0.0252 & 0.0264 & $0.0256 \pm 0.0004$ \\
\hline
\end{tabular}
finally, $\xi_{c}\left[2^{3}\right]$ is the product of two color singlets.

The dynamics of the system is governed by the Schrödinger equation

$$
\left(\mathcal{H}-E_{T}\right)|\Psi\rangle=0 \Rightarrow\left\langle\delta \Psi\left|\left(\mathcal{H}-E_{T}\right)\right| \Psi\right\rangle=0,
$$

where

TABLE II. $N N$ properties. 


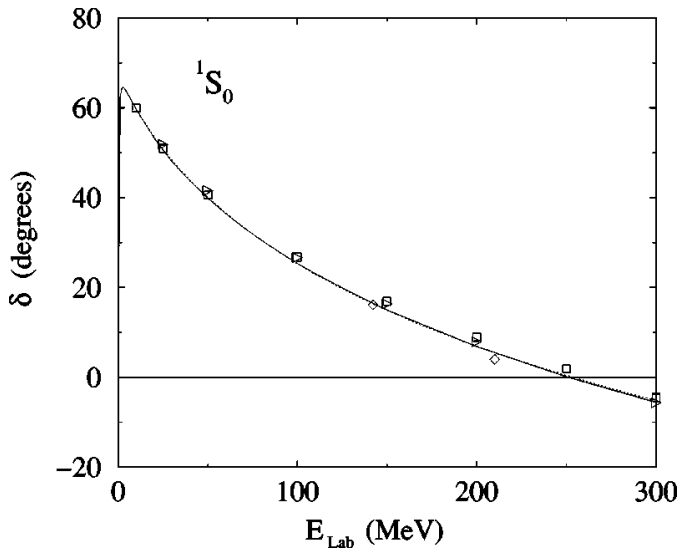

FIG. $1 .{ }^{1} S_{0} N N$ phase shift. The solid line is the result for the nonlocal quark-model potential. The dotted line shows the result of the separable representation of the nonlocal quark-model potential. The squares, diamonds, and triangles are the experimental data taken from Refs. [24], [25], and [26], respectively.

$$
\mathcal{H}=\sum_{i=1}^{N} \frac{\vec{p}_{i}^{2}}{2 m_{q}}+\sum_{i<j} V_{i j}-T_{\text {c.m. }}
$$

with $T_{\text {c.m. }}$ being the center-of-mass kinetic energy, $V_{i j}$ the quark-quark interaction described above, and $m_{q}$ the constituent quark mass.

Assuming the functional form

$$
\phi_{B}(\vec{p})=\left(\frac{b^{2}}{\pi}\right)^{3 / 4} e^{-b^{2} p^{2} / 2}
$$

where $b$ is related to the size of the nucleon quark core, Eq. (7) can be written in the following way, after the integration of the internal cluster degrees of freedom:

$$
\left(\frac{\vec{P}^{2}}{2 \mu}-E\right) \chi(\vec{P})+\int\left[V_{D}\left(\vec{P}, \vec{P}_{i}\right)+W_{L}\left(\vec{P}, \vec{P}_{i}\right)\right] \chi(\vec{P}) d \vec{P}_{i}=0
$$

$V_{D}\left(\vec{P}, \vec{P}_{i}\right)$ is the direct RGM kernel and $W_{L}\left(\vec{P}, \vec{P}_{i}\right)$ is the exchange RGM kernel, composed of three different terms

$$
W_{L}\left(\vec{P}, \vec{P}_{i}\right)=T_{L}\left(\vec{P}, \vec{P}_{i}\right)+V_{L}\left(\vec{P}, \vec{P}_{i}\right)+\left(E+E_{i n}\right) K_{L}\left(\vec{P}, \vec{P}_{i}\right),
$$

where $E_{\text {in }}$ is the internal energy of the two-body system, $T_{L}\left(\vec{P}, \vec{P}_{i}\right)$ is the kinetic energy exchange kernel, $V_{L}\left(\vec{P}, \vec{P}_{i}\right)$ is the potential energy exchange kernel, and $K_{L}\left(\vec{P}, \vec{P}_{i}\right)$ is the exchange norm kernel. Note that if we do not mind how

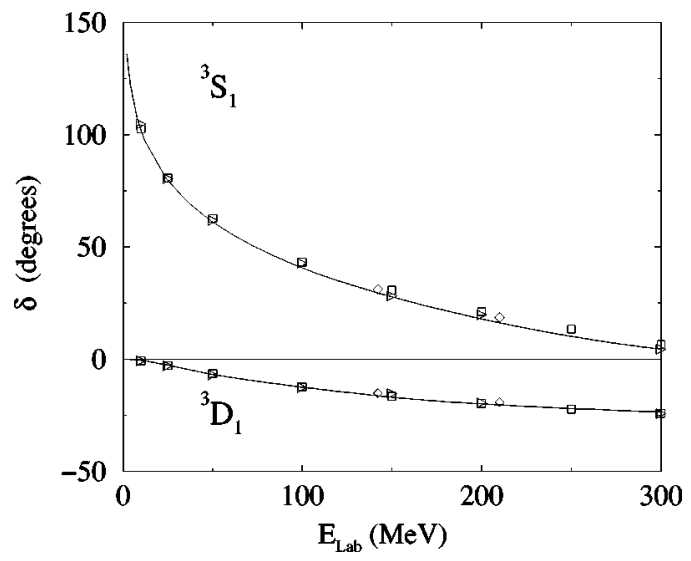

FIG. 2. Phase shifts for the ${ }^{3} S_{1}$ and ${ }^{3} D_{1}$ partial waves. Same description as in Fig. 1 except for the dotted line which is not shown.

$V_{D}\left(\vec{P}, \vec{P}_{i}\right)$ and $W_{L}\left(\vec{P}, \vec{P}_{i}\right)$ were derived microscopically, Eq. (10) can be regarded as a general single-channel equation of motion with including energy-dependent nonlocal potential. $V_{D}\left(\vec{P}, \vec{P}_{i}\right)$, which contains the direct RGM potential, and $W_{L}\left(\vec{P}^{\prime}, \vec{P}_{i}\right)$, which contains the exchange RGM potential coming from quark antisymmetry, constitute our energydependent nonlocal potential. In our case $E_{\text {in }}=2 m_{N}$ what makes our potential almost energy independent, because the center-of-mass energy of the two-body system, $E$, is much smaller than the internal energy $E_{i n}$.

The potential yields a fairly good reproduction of the experimental data up to laboratory energies of $250 \mathrm{MeV}$. For a correct description of the ${ }^{1} S_{0}$ phase shift it is necessary to take into account the coupling to the ${ }^{5} D_{0} N \Delta$ channel [17], which provides an isospin-dependent mechanism generating the additional attraction in this channel. This is implemented in our calculation generalizing Eq. (10) to a coupled channel scheme. It implies a modification of Eq. (11) with an additional term which contains the $N N \rightarrow N \Delta$ coupling. The parameters used are summarized in Table I. In Table II we present the results for the low-energy scattering data and the deuteron properties of the present model together with values of some standard $N N$ potentials [21,22] and experimental data. It is known that a charge symmetry breaking term should be included in the interaction if one wants to reproduce those quantities simultaneously [23]. This is taken into account by a slight modification of the chiral coupling constant to reproduce the deuteron and the low-energy scattering parameters (see Table I). We also show, in Figs. 1, 2, and 3, the ${ }^{1} S_{0}$ and ${ }^{3} S_{1^{-}}{ }^{3} D_{1}$ phase shifts and the mixing parameter $\varepsilon_{1}$ in comparison to results from phase-shift analyses [24$26]$.

TABLE III. Expansion (lab) energies $E_{\mu}$ (in MeV) used in the EST representations of the quark-model potential. $\epsilon_{d}$ refers to the deuteron binding energy. $l_{\mu}$ is the boundary condition chosen for the angular momentum $l_{\mu}$ of the initial state [30,31].

\begin{tabular}{lcccccc}
\hline \hline Partial wave & \multicolumn{5}{c}{$\left(E_{\mu}, l_{\mu}\right)$} \\
\hline${ }^{1} S_{0}(N N)-{ }^{5} D_{0}(N \Delta)$ & $(0,0)$ & $(50,0)$ & $(300,0)$ & $(-20,0)$ & $(-20,2)$ & $(-50,0)$ \\
${ }^{3} S_{1^{-}}{ }^{3} D_{1}$ & $\epsilon_{d}$ & $(100,0)$ & $(175,2)$ & $(300,2)$ & $(-50,0)$ & $(-50,2)$ \\
\hline \hline
\end{tabular}




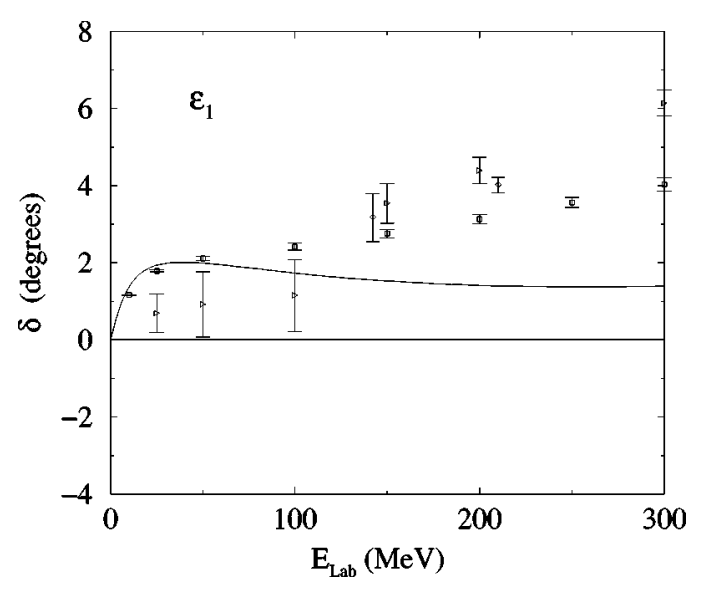

FIG. 3. Mixing parameter $\varepsilon_{1}$. Same description as in Fig. 2.

\section{TRITON BINDING ENERGY}

The triton binding energy is obtained by means of a Faddeev calculation using the $N N$ interaction described above. We perform a so-called five-channel calculation; i.e., we use only the ${ }^{1} S_{0}$ and ${ }^{3} S_{1^{-}}{ }^{3} D_{1} N N$ partial waves as input. Note that since in our model there is a coupling to the $N \Delta$ system, as explained above, a fully consistent calculation would require the inclusion of two more three-body channels. However, their contribution to the $3 N$ binding energy is known to be rather small [27] and therefore we neglect them for simplicity reasons.

To solve the three-body Faddeev equations in momentum space we first perform a separable finite-rank expansion of the $N N(-N \Delta)$ sector utilizing the Ernst-Shakin-Thaler (EST) method [28]. Such a technique has been extensively studied by one of the authors (J.H.) for various realistic $N N$ potentials [29] and specifically for a model that also includes a coupling to the $N \Delta$ system [30]. In those works it was shown that, with a separable expansion of sufficiently high rank, reliable and accurate results on the three-body level can be achieved. In the present case it turned out that separable representations of rank 6 - for ${ }^{1} S_{0}-\left({ }^{5} D_{0}\right)$ and for ${ }^{3} S_{1}-{ }^{3} D_{1}$ - are sufficient to get converged results. The set of energies used for the EST separable representations is listed in Table III. We refer the reader to Refs. [29-31] for technical details on the expansion method. The quality of the separable expansion on the $N N$ sector can be seen in Fig. 1, where we show phase shifts for the original nonlocal potential and for the corresponding separable expansion. Evidently, the phases are almost indistinguishable.

Results for the triton are summarized in Table IV. It is reassuring to see that the predicted triton binding energy is comparable to those obtained from conventional $N N$ potentials, such as the Bonn or Nijmegen models. Thus, our calculations show that quark-model based $N N$ interactions are definitely able to provide a realistic description of the triton. The results also give support to the use of such an interaction model for further few-body calculations. One should not forget at this point that the number of free parameters is greatly
TABLE IV. Properties of the three-nucleon bound state.

\begin{tabular}{lccc}
\hline \hline & Quark model & Nijm II & Bonn B [29] \\
\hline$E_{B}(\mathrm{MeV})$ & -7.72 & -7.65 & -8.17 \\
$P_{S}(\%)$ & 91.49 & 90.33 & 91.35 \\
$P_{S^{\prime}}(\%)$ & 1.430 & 1.339 & 1.368 \\
$P_{P}(\%)$ & 0.044 & 0.064 & 0.049 \\
$P_{D}(\%)$ & 7.033 & 8.267 & 7.235 \\
\hline \hline
\end{tabular}

reduced in quark-model based $N N$ interactions like the present one. In addition, the parameters are strongly correlated by the requirement to obtain a reasonable description of the baryon spectrum.

\section{CONCLUSIONS}

We have calculated the three-nucleon bound state problem utilizing a nonlocal $N N$ potential derived from a basic quarkquark interaction. This potential was generated by means of the resonating group method so that nonlocalities resulting from the internal structure of the nucleon were preserved. The resulting triton binding energy is comparable to those obtained from conventional $N N$ potentials.

In the calculation of the three-nucleon binding energy we have followed the traditional approach: namely, solving the Faddeev equations with nucleon degrees of freedom. Let us remark, however, that in a more fundamental approach one would impose consistency between the treatment of two- and three-nucleon systems in terms of quark degrees of freedom. That, of course, would require a derivation and solution of the corresponding three-nucleon RGM equations. In such a framework the quark structure of nucleons generates (besides the consecutive two-nucleon interactions that are summed up by the Faddeev equations) also genuine three-body forces. These forces are of short-ranged nature and they could be significant for short-distance phenomena like the highmomentum-transfer part of the charge form factor of ${ }^{3} \mathrm{He}$. Indeed, there have been attempts to explore the effects of such three-body forces on the triton binding energy. In a simple model based on a single one-gluon exchange [32] the three-body exchange kernels have been evaluated. An estimation provided in this reference suggests that those threenucleon forces could yield additional binding in the order of $0.2 \mathrm{MeV}$. If this is the case, then those effects would be still small enough to guarantee that the approach we followed in our study is sufficiently accurate for an exploratory calculation. However, one has to keep in mind that the estimation in Ref. [32] was done only in perturbation theory and by means of a zeroth-order three-nucleon wave function with a series of fitted parameters. Thus, for the future, a more refined and consistent treatment of the three-nucleon problem within the quark picture is certainly desirable in order to allow for reliable conclusions on this issue.

\section{ACKNOWLEDGMENTS}

The authors thank D. R. Entem for providing the codes used for the nonlocal interaction potential and for many use- 
ful comments. One of the authors (B.J-D.) wants to thank the hospitality of the FZ Jülich where part of this work was done. A.V. thanks the Ministerio de Educación, Cultura y Deporte of Spain for financial support through the Salvador de Madariaga program. This work was partially funded by Dirección General de Investigación Científica y Técnica (DGICYT) under Contract No. PB97-1401 and by Junta de Castilla y León under Contract No. SA-109/01.
[1] K. Shimizu, Rep. Prog. Phys. 52, 1 (1989).

[2] N. Isgur, Phys. Rev. D 62, 054026 (2000); nucl-th/0007008.

[3] F. Fernández, A. Valcarce, U. Straub, and A. Faessler, J. Phys. G 19, 2013 (1993).

[4] A. Valcarce, A. Buchmann, F. Fernández, and A. Faessler, Phys. Rev. C 50, 2246 (1995).

[5] M. Oka, K. Shimizu, and K. Yazaki, Nucl. Phys. A464, 700 (1987).

[6] Z. Y. Zhang, K. Bräuer, A. Faessler, and K. Shimizu, Nucl. Phys. A443, 557 (1985).

[7] L. Ya. Glozman and D. O. Riska, Phys. Rep. 268, 263 (1996).

[8] C. Nakamoto and H. Toki, Prog. Theor. Phys. 99, 1001 (1998).

[9] H. Garcilazo, F. Fernández, A. Valcarce, and R. D. Mota, Phys. Rev. C 56, 84 (1997); R. D. Mota, A. Valcarce, F. Fernández, and H. Garcilazo, ibid. 59, 46 (1999); H. Garcilazo, A. Valcarce, and F. Fernández, ibid. 60, 044002 (1999); A. Valcarce, H. Garcilazo, R. D. Mota, and F. Fernández, J. Phys. G 27, L1 (2001).

[10] S. Takeuchi, T. Cheon, and E. F. Redish, Phys. Lett. B 280, 175 (1992).

[11] B. F. Gibson, H. Kohlhoff, and H. V. von Geramb, Phys. Rev. C 51, R465 (1995).

[12] J. Haidenbauer and K. Holinde, Phys. Rev. C 53, R25 (1996).

[13] R. Machleidt, F. Sammarruca, and Y. Song, Phys. Rev. C 53, R1483 (1996).

[14] Ch. Elster, E. E. Evans, H. Kamada, and W. Glöckle, FewBody Syst. 21, 25 (1996).

[15] P. Doleschall and I. Borbély, Phys. Rev. C 62, 054004 (2000).
[16] E. F. Redish and K. Stricker-Bauer, Phys. Lett. 133B, 1 (1983).

[17] A. Valcarce, A. Faessler, and F. Fernández, Phys. Lett. B 345, 367 (1995).

[18] D. R. Entem, F. Fernández, and A. Valcarce, Phys. Rev. C 62, 034002 (2000).

[19] E. M. Henley and G. A. Miller, Phys. Lett. B 251, 453 (1991).

[20] A. de Rújula, H. Georgi, and S. L. Glashow, Phys. Rev. D 12, 147 (1975).

[21] R. Machleidt, Adv. Nucl. Phys. 19, 189 (1989).

[22] V. G. J. Stoks, R. A. M. Klomp, C. P. F. Terheggen, and J. J. de Swart, Phys. Rev. C 49, 2950 (1994); J. J. de Swart, C. P. F. Terheggen, and V. G. J. Stoks, nucl-th/9509032.

[23] D. R. Entem, F. Fernández, and A. Valcarce, Phys. Lett. B 463, 153 (1999).

[24] V. G. J. Stoks et al., Phys. Rev. C 48, 792 (1993).

[25] D. V. Bugg and R. A. Bryan, Nucl. Phys. A540, 449 (1992).

[26] R. A. Arndt, J. S. Hyslop, and L. D. Roper, Phys. Rev. D 35, 128 (1987).

[27] Ch. Hajduk and P. U. Sauer, Nucl. Phys. A322, 329 (1979).

[28] D. J. Ernst, C. M. Shakin, and R. M. Thaler, Phys. Rev. C 8, 507 (1973).

[29] W. Schadow, W. Sandhas, J. Haidenbauer, and A. Nogga, FewBody Syst. 28, 241 (2000)

[30] S. Nemoto, K. Chmielewski, N. W. Schellingerhout, J. Haidenbauer, S. Oryu, and P. U. Sauer, Few-Body Syst. 24, 213 (1998).

[31] J. Haidenbauer and W. Plessas, Phys. Rev. C 30, 1822 (1984).

[32] Y. Suzuki and K. T. Hecht, Phys. Rev. C 29, 1586 (1984). 